\title{
INFERRING CAUSAL COMPLEXITY
}

\author{
Michael Baumgartner \\ University of Bern \\ baumgartner@philo.unibe.ch
}

\begin{abstract}
In The Comparative Method Ragin (1987) has outlined a procedure of Boolean causal reasoning operating on pure coincidence data that has meanwhile become widely known as $Q C A$ (Qualitative Comparative Analysis) among social scientists. $Q C A$-also in its recent form as presented in Ragin (2000) - is designed to analyze causal structures featuring one effect and a possibly complex configuration of mutually independent direct causes of that effect. The paper at hand presents a procedure of causal reasoning that operates on the same type of empirical data as $Q C A$ and that implements Boolean techniques related to the ones resorted to by $Q C A$, yet, in contrast to $Q C A$, the procedure introduced here successfully identifies causal structures involving both mutually dependent causes, i.e. causal chains, and multiple effects, i.e. epiphenomena. In this sense, the paper at hand generalizes $Q C A$.
\end{abstract}

\section{Introduction}

In The Comparative Method Ragin (1987) has developed a methodology of causal analysis that has meanwhile become known as $Q C A$ (Qualitative Comparative Analysis) among social scientists. Ragin has introduced $Q C A$ as an alternative to standard quantitative and qualitative methodologies prevalent in social sciences. Social scientists are often confronted with data sets that are too small and too inhomogeneous for a significant statistical analyzability, yet, at the same time, are too large and too complex for an in depth qualitative analysis. $Q C A$, accordingly, is thus designed that it occupies a middle ground between the variable-oriented and the case-oriented tradition. $Q C A$ treats single cases in its data sets as complex configurations of dichotomous variables. Cases feature one dependent (effect) variable and an arbitrary amount of independent (possible cause) variables. By a systematic comparison - implementing Boolean techniques - of such configurations conjunctions of the independent variables can be identified as complex causes of the dependent variable. Every dependent variable can have several alternative complex causes which are disjunctively concatenated in the output of $Q C A$. Complex causes are seen as sufficient conditions, disjunctions of alternative causes as necessary conditions of their effects. The Boolean techniques are primarily resorted to in order to minimize complex conditions involving redundant variables.

Dichotomous variables correspond to conventional crisp sets. In Ragin (2000) $Q C A$ has been adapted for fuzzy sets, yet the fundamental presumptions of $Q C A$ 


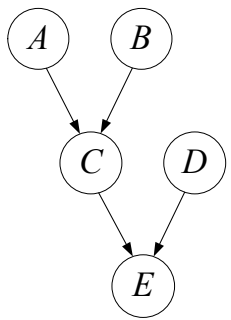

(a)

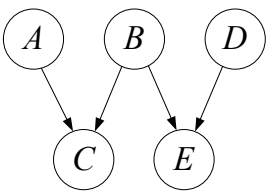

(b)

Fig. 1: Causal chains as depicted in graph (a) and epiphenomena as in (b) cannot be directly analyzed by $Q C A$. (Edges in these graphs can be seen to represent the relation of direct causal relevance.)

and, most of all, the form of causal structures uncoverable by $Q C A$ have remained unaltered. $Q C A$ is designed to analyze causal structures featuring one effect and a possibly complex configuration of mutually independent direct causes of that effect. For brevity, call the assumed singularity of the analyzed effect the singularity assumption, or ( $\mathrm{SNG}$ ) for short, and the assumed mutual independence of causes the independence assumption, or (IND) for short. Furthermore, it must be noted that an application of $Q C A$ always presupposes that it be known what variable within the set of variables to be analyzed is the effect and, accordingly, what variables are possible causes. I shall refer to the assumed identifiability of causes and effects as (ICE). ${ }^{1}$

Certain ubiquitous causal structures violate (SNG) and (IND), most notably causal chains and epiphenomena. Examples for both of these structures are graphed in figure 1. Of course, a subdivision of a chain as the one in graph 1(a) into its separate layers $-A \vee B \Rightarrow C$ and $C \vee D \Rightarrow E$, respectively ${ }^{2}$ - yields two causal substructures that satisfy both (SNG) and (IND). Such a subdivision would thus render a chainlike structure amenable to a stepwise $Q C A$ analysis: First $A \vee B \Rightarrow$ $C$ and then $C \vee D \Rightarrow E$ could be uncovered by means of $Q C A$. An analogous subdivision of the epiphenomenon in 1(b) would render that structure modularly tractable by $Q C A$. However, such a breaking down of complex structures into simple ones that satisfy ( $\mathrm{SNG}$ ) and (IND) presupposes that a great deal about the causal structure under investigation be known prior to its analyzability by $Q C A-$ hence (ICE). The variables involved in the investigated cases must be categorized into possible causes and possible effects prior to implementing $Q C A$. In the end, what $Q C A$ determines is whether possible causal dependencies in fact exist and

\footnotetext{
${ }^{1}$ It shall not be claimed that these 3 assumptions are logically independent. They are just labelled here for the purpose of easy reference later on. Moreover, it must be pointed out that (SNG), (IND) and (ICE) are not explicitly assumed in the context of $Q C A$, rather they are implicitly taken for granted.

${ }^{2}$ The " $\Rightarrow$ ” operator is given a specific interpretation below. For now, it can just be understood in terms of the causal relation.
} 
whether the cause variables constitute complex or alternative causes of the effect under investigation.

$Q C A$ draws on concepts and ideas developed within the regularity theoretic tradition of the philosophy of causation. Ragin himself sees $Q C A$ as a generalization and systematization of Mill's methods of agreement and difference, and the core of the Boolean techniques to minimize causal conditions implemented in QCA can be found in Broad (1930), Broad (1944), or Mackie (1974). ${ }^{3}$ The paper at hand takes on to show that prior knowledge about the causal structure under investigation does not need to be presupposed against a regularity theoretic background. The latter allows for causal reasoning without presuming (SNG) and (IND). Thus, in what follows I shall present a procedure of causal reasoning that processes the same kind of empirical data as $Q C A$, implements Boolean techniques closely related to the ones resorted to by $Q C A$, yet, in contrast to $Q C A$, does neither presuppose (SNG) nor (IND) nor (ICE).

\section{The Background}

As mentioned above, the theoretical background of the inference procedure to be developed in this paper is located in the regularity theoretic tradition of the philosophy of causation. The core of this background shall be very briefly reviewed before the procedure is introduced. ${ }^{4}$ Regularity theories of causation analyze causes and effects on type-level, i.e. event types - or factors for short - are seen as the primary relata of the causal relation. The primary analysans of a regularity theory, hence, is general causation. A factor that causes another factor is said to be causally relevant to the latter. Factors are taken to be similarity sets of event tokens. They are sets of type identical token events, of events that share at least one feature. Contrary to token events, event types are generic entities. They are not located in time and space themselves, but they are instantiated in time and space by token events. Whenever a member of a similarity set that corresponds to an event type occurs, the latter is said to be instantiated.

Factors are symbolized by italicized capital letters $A, B, \ldots, H, H_{1}, H_{2}$ etc., with variables $Z, Z_{1}, Z_{2}$ etc. running over the domain of factors. An event type as "peasant revolt" $(A)$ can be defined as the set consisting of all token events in the extension of the predicate " $\ldots$ is a peasant revolt", i.e. as $\{x: x$ is a peasant revolt $\}$. Factors are negatable. The negation of a factor $A$ is written thus: $\bar{A}$. $\bar{A}$ is simply defined as the complementary set of $A$.

Causal analyses are always relativized to a set of investigated factors. This set is referred to as the factor frame of a causal investigation. Factors are virtually never causally relevant to their effects in isolation. Rather, they are parts of whole causing complexes - complex causes. A complex cause only becomes causally

\footnotetext{
${ }^{3}$ Cf. also Quine (1959).

${ }^{4}$ For details on the theoretical background resorted to here see Baumgartner (forthcoming), Baumgartner and Graßhoff (2004) and Graßhoff and May (2001).
} 
effective if all of its constituents are co-instantiated, i.e. instantiated close-by or coincidently. Coincidently instantiated factors are termed coincidences. As will be shown below, coincidences constitute the empirical data processed by the inference procedure to be developed in this paper. ${ }^{5}$

Essentially, modern regularity theories analyze causal relevance with recourse to minimalized regularities among factors. The crucial notion needed in the definiens of causal relevance is the notion of a minimal theory. Briefly, a minimal theory of a factor $B$ is a minimally necessary disjunction of minimally sufficient conditions of $B$. A conjunction of coincidently instantiated factors $A_{1} \wedge A_{2} \wedge \ldots \wedge A_{n}$, which for simplicity shall be abbreviated by a mere concatenation of the respective factors, is a minimally sufficient condition of a factor $B$ iff $A_{1} A_{2} \ldots A_{n}$ is sufficient for $B$, i.e. $A_{1} A_{2} \ldots A_{n} \rightarrow B$, and there is no proper part $\alpha$ of $A_{1} A_{2} \ldots A_{n}$ such that $\alpha \rightarrow B$. A "proper part" of a conjunction designates the result of any reduction of this conjunction by one conjunct. Analogously, a disjunction of factors $A_{1} \vee A_{2} \vee \ldots \vee A_{n}$ is a minimally necessary condition of a factor $B$ iff $A_{1} \vee A_{2} \vee \ldots \vee A_{n}$ is necessary for $B$, i.e. $B \rightarrow A_{1} \vee A_{2} \vee \ldots \vee A_{n}$, and there is no proper part $\beta$ of $A_{1} \vee A_{2} \vee \ldots \vee A_{n}$ such that $B \rightarrow \beta$. A "proper part" of a disjunction designates the result of any reduction of this disjunction by one disjunct.

That a disjunction of minimally sufficient conditions of a factor $B$ is minimally necessary for $B$ shall be symbolized by ' $\Rightarrow$ ' which is termed a double-conditional. Thus, a minimal theory has the following double-conditional form:

$$
A C \vee D E \vee F G H \Rightarrow B
$$

Informally, (1) says that whenever $A C$ or $D E$ or $F G H$ are instantiated, $B$ is instantiated as well, and whenever $B$ is instantiated $A C$ or $D E$ or $F G H$ is instantiated as well. In this vein, both the principle of determinism and the principle of causality are formally captured in a straightforward way: Causes determine their effects and if no causes are present, the effect is not present either. ${ }^{6}$ Membership in a minimal theory induces direct causal relevance: A factor $A$ is directly causally relevant to a factor $B$ iff $A$ is part of a minimal theory of $B$, i.e. iff $A$ is a non-redundant part of a minimally sufficient condition of $B$ which, in turn, is a non-redundant part of a minimally necessary condition of $B .{ }^{7}$ Hence, (1) represents a causal structure such that $A C, D E$ and $F G H$ are alternative complex causes of $B$.

\footnotetext{
${ }^{5}$ Coincidences correspond to what Ragin (1987) calls configurations.

${ }^{6}$ The question as to whether the causal relation in fact is deterministic or not shall be bypassed here. Whoever holds that there are irreducibly indeterministic causal processes, can simply view regularity theories as analyses of the deterministic variant of causal processes.

${ }^{7}$ In fact, in order for a minimal theory $\Phi$ to be causally interpretable, certain relational constraints, as spatiotemporal proximity, have to be imposed on the events that instantiate the factors in $\Phi$. For simplicity, these constraints are neglected in the present context. For a detailed presentation of the logical form of minimal theories cf. Baumgartner (forthcoming). Furthermore, as section 10 below shows, minimalizing necessary conditions paves the way for an accurate regularity theoretic treatment of epiphenomena, which - on account of Mackie's (1974) famous Manchester Factory Hooters counterexample - have often been considered intractable by regularity theories.
} 
Analyzing the disjunction of alternative causes of $B$ as necessary condition of $B$ amounts to claiming sufficiency of $B$ for just that disjunction. As is often done by critics of regularity accounts, the question might thus be raised as to how the above analysis of causal relevance captures the undisputed asymmetry of that relation. For if $B$ can be shown to be minimally sufficient for $A C \vee D E \vee F G H$ it might be argued that - relative to the above analysis of causal relevance $-B$ is likewise to be considered causally relevant to its alternative causes. Contrary to first appearances, however, double-conditionals as (1) are not symmetrical with respect to the expressions to the left and the right of " $\Rightarrow$ ". The instantiation of a particular disjunct is minimally sufficient for $B$, but not vice versa. $B$ does not determine a particular disjunct to be instantiated. ${ }^{8} B$ only determines the whole disjunction of minimally sufficient conditions. $A C$ and $D E$ and $F G H$ are each minimally sufficient for $B$, the latter however is only minimally sufficient for $A C \vee D E \vee$ $F G H$. Hence, given that an instantiation of $A C$ is observed, it can be inferred that there also is an instance of $B$. On the other hand, if an instance of $B$ is observed, no such inference to an instantiation of $A C$ is possible. The observed instance of $B$ might well have been caused by $D E$. This asymmetry corresponds to the asymmetry of determination.

Accounting for the asymmetry of causal relevance in this vein has an important implication as regards the minimal complexity of causal structures. A condition $A C$, that is both minimally sufficient and necessary for a factor $B$, cannot be identified as cause of $B$, for $B$ would be minimally sufficient and necessary for $A C$ as well. All empirical evidence such a dependency structure would generate are perfectly correlated instantiations of $A C$ and $B$ - both would either be co-instantiated or absent. Such empirical data could only be causally interpreted if external asymmetries - as e.g. temporal order - holding among the instances of $A C$ and $B$ would be available. However, as the procedure of causal reasoning to be presented in this paper shall infer causal structures on the same empirical basis as $Q C A$, i.e. on the basis of mere coincidence information, perfect correlations among factors shall be taken not to be causally interpretable in the present context. In order to distinguish causes from effects and to orient the cause-effect relation based on coincidence information alone, at least two alternative causes are needed for each effect. ${ }^{9}$

Ordinary causal structures far exceed (1) in complexity. Most causally relevant factors are of no interest to causal investigations or are unknown. That is why minimal theories either need to be relativized to a given causal background or must be kept open for later extensions. The latter is achieved by means of variables. Variables $X_{1}, X_{2}, \ldots$ are introduced to stand for an open number of additional conjuncts within a sufficient condition, while $Y_{A}, Y_{B}, \ldots$ are taken to stand for an open number of additional disjuncts in a minimal theory. If (1) is in this sense kept

\footnotetext{
${ }^{8}$ Cf. Graßhoff and May (2001), pp. 97-99. Similar analyses of the direction of causation have been proposed in Sanford (1976), Ehring (1982), and Hausman (1998).

${ }^{9} Q C A$ does not face the problem of the orientation of causal dependencies, for applying $Q C A$ is taken to be possible only if the effect has been identified within the analyzed factor frame (cf. (ICE)). As (ICE) shall be given up here, however, a way to orient dependencies among factors is needed.
} 
open for additional factors, one gets:

$$
A C X_{1} \vee D E X_{2} \vee F G H X_{3} \vee Y_{B} \Rightarrow B
$$

However, as will be shown below causal inferences drawn from coincidence data always must be relativized to the homogeneity of the causal background of that data. Of course, relative to a given background two or three factors may well be sufficient for an effect as indicated in (1). Thus, either a minimal theory as (1) is explicitly relativized to a causal background or it is rendered background independent by means of variables as in (2). Both ways of doing justice to the openness of causal structures will be implemented below as convenient.

While direct causal relevance is analyzed with recourse to membership in simple minimal theories as (1), complex causal structures as causal chains or epiphenomena are represented by complex minimal theories. Simple minimal theories can be conjunctively concatenated to complex theories: A conjunction of two minimal theories $\Phi$ and $\Psi$ is a complex minimal theory iff, first, at least one factor in $\Phi$ is part of $\Psi$ and, second, $\Phi$ and $\Psi$ do not have an identical consequent. ${ }^{10}$ The following are two complex minimal theories:

$$
\begin{aligned}
& \left(A X_{1} \vee D X_{2} \vee Y_{B} \Rightarrow B\right) \wedge\left(B X_{4} \vee G X_{5} \vee Y_{H} \Rightarrow H\right) \\
& \left(A X_{1} \vee D X_{2} \vee Y_{B} \Rightarrow B\right) \wedge\left(D X_{4} \vee G X_{5} \vee Y_{H} \Rightarrow H\right)
\end{aligned}
$$

(3) represents a causal chain $-B$ is the effect factor of the first conjunct and a cause factor in the second conjunct-, (4) stands for an epiphenomenon - $D$ is a common cause of $B$ and $H$. In this vein, causal structures of arbitrary complexity can be represented on regularity theoretic grounds. Accordingly, a factor $A$ can be said to be indirectly causally relevant to a factor $B$ iff there is a sequence of factors $Z_{1}$, $Z_{2}, \ldots, Z_{n}, n \geq 3$, such that $A=Z_{1}, B=Z_{n}$, and for each $i, 1 \leq i<n: Z_{i}$ is part of the antecedent of a simple minimal theory of $Z_{i+1}$.

\section{The Basic Idea and Input Data}

Minimal theories represent causal structures in a transparent way. Conjunctions in the antecedent of a minimal theory stand for complex causes of the factor in the consequent, disjunctions for alternative causes. Hence, minimal theories are directly causally interpretable. Moreover, minimal theories impose constraints on the behavior of the factors contained in them. For instance, (1) says that whenever $A C$ is instantiated, there also is an instance of $B$. That means, according to (1) the coincidence $A C \bar{B}$ does not occur. Correspondingly, information about occurring and non-occurring coincidences allows for conclusions as to the minimal

\footnotetext{
${ }^{10}$ The first constraint guarantees that complex minimal theories represent cohering causal structures and the second restriction prohibits the conjunctive concatenation of equivalent minimal theories and thus excludes redundancies. Again, relational constraints - as spatiotemporal proximity imposed on the instances of complex minimal theories are neglected here.
} 


\begin{tabular}{ccc}
$A$ & $B$ & $C$ \\
\hline 1 & 1 & 1 \\
1 & 0 & 1 \\
0 & 1 & 1 \\
0 & 0 & 0
\end{tabular}

(a)

\begin{tabular}{ccc}
$A$ & $B$ & $C$ \\
\hline 1 & 1 & 1 \\
1 & 1 & 0 \\
0 & 1 & 1 \\
1 & 0 & 1 \\
1 & 0 & 0 \\
0 & 1 & 0 \\
0 & 0 & 1 \\
0 & 0 & 0
\end{tabular}

(b)

\begin{tabular}{ccc}
$A$ & $B$ & $C$ \\
\hline 1 & 1 & 1 \\
0 & 1 & 1 \\
1 & 0 & 1 \\
1 & 0 & 0 \\
0 & 1 & 0 \\
0 & 0 & 1 \\
0 & 0 & 0
\end{tabular}

(c)

\begin{tabular}{ccc}
$A$ & $B$ & $C$ \\
\hline 1 & 1 & 1 \\
0 & 0 & 0
\end{tabular}

(d)

Tab. 1: Simple examples of coincidence lists as processed by CA.

theory representing the underlying causal structure. If it is known that $A C$ is never realized in combination with $\bar{B}$, while both $A \overline{C B}$ and $\bar{A} C \bar{B}$ are found to be empirically possible, it follows that $A C$ is minimally sufficient for $B$. In this sense, minimal theories constitute the link between the empirical behavior of the factors in an investigated frame and the causal structure behind that behavior. The empirical behavior of the factors allows for inferring minimal theories that describe that behavior, and these minimal theories, in turn, are causally interpretable.

The procedure of causal reasoning to be developed here operates on the same data as $Q C A$ : coincidences of the factors involved in a causal process whose structure is to be revealed. Accordingly, the procedure shall be termed coincidence analysis or CA for short. Contrary to $Q C A$, however, the data fed into CA is not required to mark one factor as the effect. Based on its input data, CA simply determines for each factor $Z_{i}$ in the analyzed frame involving, say, $n$ factors which dependencies hold between $Z_{i}$ and the other $n-1$ factors in the frame. Most of these dependencies will turn out not to be causally interpretable. The possibly causally interpretable dependencies are subsequently minimalized and expressed in terms of minimal theories, which, finally, are straightforwardly causally interpretable as shown above. Moreover, CA does not require the $n-1$ other factors to be independent, i.e. to be co-instantiatable in all logically possible $2^{n-1}$ combinations.

As in case of $Q C A$, the data processed by CA is listed analogously to truthtables. Tables as in 1 are referred to as coincidence lists. The rows in a coincidence list shall be numbered starting with the first row below the title row. The row constituted by " $1 \quad 1 \quad 1$ " in list (a) is row 1 (R1), the row featuring " $1 \quad 0 \quad 1$ " is row 2 (R2), and so on. In coincidence lists a ' 1 ' in the column of, say, factor $A$ represents an instance of $A$, a ' 0 ' in that same column symbolizes the absence of such an instance. Columns of coincidence lists thus record instances and absences of the factor mentioned in the title row, while the rows following the title row specify 
coincidences of the factors in the title row. For example, the first row, R1, of (a) records the coincidence $A B C$, the following row, $\mathrm{R} 2$, indicates the coincidence $A \bar{B} C$.

List (a) in table 1 clearly manifests dependencies among its factors. For instance, there is no row in (a) featuring $A B \bar{C}$. That means the coincidence $A B$ is sufficient for $C$. Likewise, there is no row in (a) featuring $A$ in combination with $\bar{C}$, which amounts to the sufficiency of $A$ for $C$. The sufficient condition $A B$, hence, contains a sufficient proper part, $A$, and, accordingly, is not minimally sufficient. Analogously it can be shown that $\bar{B} C$ is minimally sufficient for $A$ in list (a). As will be shown below, some of these dependencies are causally interpretable, others are not.

Thereagainst, list (b) contains all 8 logically possible configurations of the 3 factors in its frame. (b) is therefore referred to as a complete coincidence list: A coincidence list over a factor frame of $n$ factors is complete iff it contains all logically possible combinations of the involved factors, i.e. iff it is constituted by $2^{n}$ rows. Complete lists do not feature dependencies among their factors. No combinations of factors are sufficient or necessary for any factor in the respective frame. Accordingly, complete lists do not need to be analyzed for dependencies to begin with. Dependencies only emerge in incomplete lists, i.e. in lists that feature less than $2^{n}$ coincidences of the $n$ factors in their frame. List (c) in table 1 is incomplete in this sense. There is no row in that list such that $A$ and $B$ are instantiated without an instance of $C . A B$ is minimally sufficient for $C$ relative to list (c). Finally, list (d) is incomplete as well. It is incomplete to such an extent that too many dependencies emerge. According to list (d), every factor is minimally sufficient and necessary for every other factor in the corresponding frame. Such an abundance of dependencies is not causally interpretable, for causes and effects cannot be distinguished. As the previous section has shown, if causal dependencies are to be oriented on the basis of mere coincidence data - and not, as in case of $Q C A$, by assumption (ICE) -, at least two alternative causes are required for each effect. All of this shows, that not all coincidence lists are causally interpretable.

\section{Presuppositions}

While CA dispenses with assumptions (SNG), (IND) and (ICE), it still rests on two important presuppositions, the first of which is equally endorsed by $Q C A$ while the second is replaced by another assumption to the same effect in $Q C A$ : First, unambiguous causal inferences are only possible given that the coincidence data is exhaustive and, second, the causal background of coincidence lists must be homogeneous. Let us take these presuppositions in turn.

Any procedure of causal reasoning, in some way or another, assumes that its input data is exhaustive. Probabilistic procedures presume the availability of probability distributions over all exogenous variables, or $Q C A$ relies on the realizability 
of all $2^{n}$ configurations given $n$ independent causal variables. Nonetheless, assumptions as regards the exhaustiveness of empirical data are hardly ever made explicit in studies on causal reasoning. ${ }^{11}$ Such an implicit taking for granted of the suitability of input data, however, will not do for the present context. As the previous section has shown, dependencies among $n$ factors emerge only if not all $2^{n}$ coincidences are contained in an analyzed coincidence list. Of course, however, coincidences may not only be missing from coincidence lists due to causal dependencies among respective factors. Exhaustive data collection may fail for a host of different reasons. Financial or technical resources may happen to be limited in experimental sciences or nature may be found not to provide sufficient data in nonexperimental disciplines. Inexhaustive data is likely to be one of the main reasons for hampered causal interpretability of that data. Data collection, however, is not part of causal reasoning, but a precondition thereof. That is why (PEX) is endorsed in the present context, which is concerned with matters of causal reasoning only.

Principle of Empirical Exhaustiveness (PEX): The collection of empirical data to be processed by CA faces no practical limitations whatsoever. All coincidences of the analyzed factors that are compatible with the causal structure regulating the behavior of these factors are in fact observed.

(PEX) guarantees that whenever a coincidence is missing from a CA-processed list, this is due to underlying causal dependencies. Clearly, (PEX) constitutes a sweeping idealization with respect to data collection. Such an idealization, however, may prove to be useful in many practical contexts. It can be implemented as a gauge by means of which concrete data collections can be measured and thus evaluated. (PEX) is not a precondition of causal reasoning per se, but a precondition of unambiguous causal reasoning. Even inexhaustive data provides some information as to the underlying causal structure. For instance, in list (a) of table 1, factors $A$ and $B$ are independent. This independence will remain unaltered irrespective of further coincidences introduced into list (a). Thus, if (a) violates (PEX), there is no single causal structure that can be identified as underlying the behavior of the factors in that list. Nonetheless a set of causal structures can be determined to possibly underly the coincidences in (a): the set of all causal structures over the factor frame $\{A, B, C\}$ such that $A$ and $B$ are causally independent. Still, for reasons of unambiguity (PEX) shall be presumed in the following.

Apart from (PEX) an application of CA must assume that the causal background of an analyzed coincidence list is causally homogeneous. A list as (a) in table 1 could be generated by suitable manipulation of each factor separately. A causal interpretation of such an 'artificial' list, of course, would be fallacious. Causal relevancies would be attributed to factors in the frame which, in fact, did not contribute to the behavior of respective effect factors. Such as to forestall causal fallacies, i.e. in order for a list to reveal the underlying causal structure, it

\footnotetext{
${ }^{11}$ One exception is Ragin $(1987,2000)$. He discusses at length how limited empirical data negatively affects causal reasoning.
} 
must be presumed that the behavior of the factors in the investigated frame is not confounded by causally relevant factors not contained in the frame. Each analysis of a causal process is limited to a small subset of all factors involved in that process. Causal processes are extremely complex. Ordinarily, only a few factors are of interest in the course of concrete causal investigations. While Ragin (1987) nonetheless assumes that a $Q C A$-analyzed coincidence list contains all causally relevant factors, we shall simply presuppose that $\mathrm{CA}$-analyzed coincidence lists are generated against homogeneous backgrounds. We thus do not demand that all causally relevant factors of an investigated structure are contained in a factor frame. A coincidence list over a frame consisting of $Z_{1}, \ldots, Z_{n}$ is assumed to be homogeneous with respect to confounders not contained in $\left\{Z_{1}, \ldots, Z_{n}\right\}$. In order to spell out the notion of a confounder needed for our purposes, the notion of a causal path is required: A sequence of factors $\left\langle Z_{1}, \ldots, Z_{k}\right\rangle, k \geq 2$, constitutes a causal path from $Z_{1}$ to $Z_{k}$ iff for each $Z_{i}$ and $Z_{i+1}, 1 \leq i<k$, in the sequence: $Z_{i}$ is directly causally relevant to $Z_{i+1}$. A condition $X_{i}$ is said to be part of a causal path, if a at least one conjunct of $X_{i}$ is contained in the sequence constituting that path. Now the notion of a confounder can be clarified: If $Z_{n}$ is an effect, a confounder of $Z_{n}$ is a minimally sufficient condition $X_{i}$ of $Z_{n}$ such that $X_{i}$ is causally relevant to $Z_{n}$ and $X_{i}$ is part of a causal path leading to $Z_{n}$ not containing any of the factors $Z_{1}, \ldots, Z_{n-1}$. That means, a factor $Z_{o}$ that is causally relevant to an effect $Z_{n}$ and that is not contained in the investigated frame $\left\{Z_{1}, \ldots, Z_{n}\right\}$ cannot confound causal reasoning if all causal paths connecting $Z_{o}$ and $Z_{n}$ contain at least one factor in $\left\{Z_{1}, \ldots, Z_{n}\right\}$, i.e. if $Z_{o}$ is a cause or an effect of a factor in the investigated frame. A confounder is a factor or a conjunction of factors by means of which the investigated effect can be manipulated independently of the factors in the frame.

The notion of a confounder is to be understood relatively to a corresponding effect. Basically, any factor in an analyzed frame can be seen as effect of an underlying causal structure. However, as will be shown below, there are several constraints due to which a factor can be excluded from the set $W$ of potential effects contained within a given factor frame prior to causally analyzing that frame. Still, depending on the specific $Z_{i} \in \mathrm{W}$ analyzed in the course of a given run of CA, different factors are to be seen as confounders and, accordingly, must be homogenized. Generally: Input data processed by $C A$ is assumed to be generated against causally homogeneous backgrounds in the sense of $(\mathrm{HC})$ :

Homogeneity (HC): The background of a causally analyzed list of $m$ coincidences over a factor frame containing the set $\mathrm{W}$ of potential effects is causally homogeneous iff for every confounder $X_{i}$ of every factor in W: $X_{i}$ is absent in the background of one coincidence iff $X_{i}$ is absent in the backgrounds of all other $m-1$ coincidences.

While only homogeneous coincidence lists are causally analyzable, (HC) does not guarantee the causal analyzability of coincidence lists. Rather, (HC) prevents causal fallacies. Therefore, a coincidence list may well be homogeneous in terms of 


\begin{tabular}{ccc}
$A$ & $B$ & $C$ \\
\hline 1 & 1 & 1 \\
1 & 0 & 1 \\
0 & 1 & 1 \\
1 & 1 & 0
\end{tabular}

(a)

\begin{tabular}{ccc}
$A$ & $B$ & $C$ \\
\hline 1 & 0 & 0 \\
0 & 1 & 0 \\
0 & 0 & 1 \\
0 & 0 & 0
\end{tabular}

(b)

Tab. 2: Two coincidence lists that cannot be causally analyzed, for none of the involved factors can be interpreted as effect of an underlying causal structure in accordance with (HC).

(HC), even though confounders are instantiated in its background - as long as these confounders are instantiated in the backgrounds of all coincidences. If confounders are universally instantiated, effects will be present in all coincidences, irrespective of whether the other factors in the frame are present or absent. In this case no dependencies emerge and thus no inferences as to underlying causal structures are drawn. As a consequence no causal fallacies are committed either.

(HC) excludes a number of coincidence lists from causal analyzability. The lists fed into CA may well reveal certain backgrounds to be causally inhomogeneous. Consider, for instance, the lists in table 2. Assume $B$ to be an effect of the causal structure generating list (a) in table 2. A comparison of the test situations recorded in row 1 (R1) and 2 (R2) of that list shows that, if $B$ in fact were the effect of the underlying structure, the test situations recorded in (a) would violate (HC). The only factor varying in $\mathrm{R} 1$ and $\mathrm{R} 2$ is $B$; no other factor in the frame $\{A, B, C\}$ is accountable for that variation of $B$, therefore it must be due to a varying confounder of $B$ in the unknown or unconsidered background of list (a). That means, assuming $B$ to be an effect contradicts the homogeneity assumption. If $B$ is taken to be a cause factor of the underlying structure, (HC) is not violated. Thus, assuming (HC) to hold for list (a) implies that $B$ cannot be seen as a possible effect. The same holds for the other two factors in $\{A, B, C\}$. In R1 and R3 $A$ is the only varying factor, while no other factor, apart from $C$, varies in $\mathrm{R} 1$ and $\mathrm{R} 4$. Hence, there is no factor in list (a) that could possibly be an effect of an underlying causal structure in accordance with (HC). Analogous considerations apply to list (b). In $\mathrm{R} 1$ and $\mathrm{R} 4$ of that list $A$ is the only varying factor, $\mathrm{R} 2$ and $\mathrm{R} 4$ exclude $B$ from being interpretable as an effect, and $\mathrm{R} 3$ and $\mathrm{R} 4$ refuse $C$ admittance into the set of possible effects due a violation of $(\mathrm{HC})$.

That means, there cannot be a causal structure underlying either list (a) or (b) that would be compatible with (HC). In neither list there is a factor that could be seen as an effect in accordance with (HC), i.e. $W=\emptyset$. Whenever for every factor $Z_{i}$ contained in the factor frame of a coincidence list $\mathcal{C}$ there are two rows $\mathrm{R} k$ and $\mathrm{R} l$ in $\mathcal{C}$ such that $Z_{i}$ is the only factor varying in $\mathrm{R} k$ and $\mathrm{R} l$, the background against which the data in $\mathcal{C}$ is collected cannot be homogeneous, for there is no causal structure that could possibly generate $\mathcal{C}$ and accord with (HC). We shall 
in this context speak of inhomogeneous coincidence lists. (HC) excludes all inhomogeneous coincidence lists from being processed by CA. It must be emphasized, however, that the homogeneity of coincidence lists is an assumption to which every inference of CA must be relativized. It might well be that a coincidence list which is not inhomogeneous in the sense defined above, as e.g. list (a) in table 1, in fact is the result of an uncontrolled variation of background confounders. In this sense, only a sufficient and no necessary condition for the inhomogeneity of a coincidence list is given above. Causal inferences drawn by CA will always be of the form "Given that (HC) is satisfied, such and such must be the underlying causal structure". Homogeneity is never beyond doubt. Nonetheless, assembling the theoretic preconditions of conclusive causal inferences serves the goal of establishing standards implementable as a gauge for concrete causal analyses.

\section{Identification of Potential Effects}

After having clarified the presuppositions on which CA rests, we now proceed to introduce the inference rules of CA. As anticipated in the previous section, a first algorithmic step consists in parsing through the factor frame of a coincidence list in order to determine which of the factors could possibly operate as effects within the causal structure to be revealed. This step yields a set $W$ of factors whose dependencies on the other factors in the corresponding frame are then successively determined by CA. The identification of potential effects shall not be considered a proper part of CA, for any sort of context dependent empirical information or even prior causal knowledge is allowed to enter the determination of W. For instance, if a factor $Z_{i}$ is generally instantiated temporally before every other factor in an analyzed frame $\left\{Z_{1}, \ldots, Z_{n}\right\}, Z_{i}$ cannot function as an effect within the underlying causal structure. Or prior causal knowledge could be available that establishes the members of a proper subset of $\left\{Z_{1}, \ldots, Z_{n}\right\}$ as root factors, i.e. as factors that are causes, but no effects within a causal structure. In both cases there is no need to integrate respective factors in W. CA does not have to evaluate dependencies among factors that can be excluded from the set of potential effects to begin with. These pragmatic circumstances are not systematizable or, at least, a systematization shall not be attempted here. Accordingly, no recursively applicable or computable rule can be provided, which essentially is why the determination of $W$ is not seen as a proper part of CA.

Still, the determination of $\mathrm{W}$ is not only regulated by spatiotemporal peculiarities of an analyzed process or by prior causal knowledge. As the previous section has shown, factors can be excluded from the set of potential effects based on homogeneity considerations. Backgrounds of coincidences are assumed to be homogeneous in terms of (HC). Now, if a factor $Z_{i}$ is the only factor varying in two coincidences contained in an analyzed list and if, moreover, $Z_{i}$ were seen as a potential effect of the underlying causal structure, $(\mathrm{HC})$ would be violated with respect to $Z_{i}$. Therefore, in order for a factor $Z_{i}$ to be a potential effect, it must not 
be the case that the corresponding coincidence list contains two rows such that $Z_{i}$ is the only varying factor in those rows.

Furthermore, since CA shall be designed to infer causes of both positive and negative factors, $\mathrm{W}$, in principle, may contain both positive and negative factors. However, to every minimal theory of a positive factor $Z_{i}$, there exists an equivalent minimal theory of $\overline{Z_{i}}$, and vice versa.

$$
\begin{array}{r}
A C \vee D E \Rightarrow B \\
\overline{A D} \vee \overline{A E} \vee \overline{C D} \vee \overline{C E} \Rightarrow \bar{B}
\end{array}
$$

(5) and (6) are logically equivalent and one of these expressions is a minimal theory if and only if the other one is too. ${ }^{12}$ Hence, for simplicity's sake, CA can be confined to identify minimal theories of either positive factors or their negative counterparts. For this reason, we stipulate that positive factors only shall be included in W.

These considerations taken together yield the following standard as regards the determination of $\mathrm{W}$. In order to indicate that the non-computable identification of the set of potential effects is a precondition of launching CA, yet not a proper part thereof, it shall be referred to as "step $0 *$ ".

Step $0^{*}$ - Identification of potential effects: Given a coincidence list $\mathcal{C}$ over a factor frame $\left\{Z_{1}, \ldots, Z_{n}\right\}$, identify the subset $\mathrm{W} \subseteq\left\{Z_{1}, \ldots, Z_{n}\right\}$ such that for every $Z_{i}: Z_{i} \in \mathrm{W}$ iff

(1) The totality of available information as to the spatiotemporal ordering of the instances of the factors in $\left\{Z_{1}, \ldots, Z_{n}\right\}$ and the available prior causal knowledge about the behavior of the factors in $\left\{Z_{1}, \ldots, Z_{n}\right\}$ does not preclude $Z_{i}$ to be an effect of the underlying causal structure.

(2) $\mathcal{C}$ does not contain two rows $\mathrm{R} k$ and $\mathrm{R} l$ such that $Z_{i}$ is the only factor varying in the coincidences recorded by $\mathrm{R} k$ and $\mathrm{R} l$.

(3) $Z_{i}$ is a positive factor.

\section{Identification and Minimalization of Sufficient Conditions}

Upon having identified a non-empty set of potential effects, CA proper sets in. In a first stage, sufficient conditions for each member of $W$ are identified and minimalized. In order to illustrate this first stage, let us look at a concrete example. Assume the coincidence list depicted in table 3 to be our input data. None of the factors in our exemplary frame $\{A, B, C, D, E\}$ shall be excluded from effect position by prior causal knowledge or additional information as to the spatiotemporal ordering of the instances of these factors. Nonetheless, the set of potential effects does not correspond to the factor frame of table 3, i.e. $\mathbf{W} \neq\{A, B, C, D, E\}$. For reasons of

\footnotetext{
${ }^{12}$ For a detailed proof of the existence of an equivalent minimal theory of a negative factor to every minimal theory of a positive factor cf. Baumgartner (forthcoming), ch. 3.
} 


\begin{tabular}{ccccc}
$A$ & $B$ & $C$ & $D$ & $E$ \\
\hline 1 & 1 & 1 & 1 & 1 \\
1 & 1 & 1 & 0 & 1 \\
1 & 0 & 1 & 1 & 1 \\
1 & 0 & 1 & 0 & 1 \\
0 & 1 & 1 & 1 & 1 \\
0 & 1 & 1 & 0 & 1 \\
0 & 0 & 0 & 1 & 1 \\
0 & 0 & 0 & 0 & 0
\end{tabular}

Tab. 3: Exemplary coincidence list to be analyzed by CA.

compatibility with (HC), factors $A, B$, and $D$ cannot be effects. For each of these factors there is a pair of rows in table $3-\langle\mathrm{R} 1, \mathrm{R} 5\rangle$ for $A,\langle\mathrm{R} 1, \mathrm{R} 3\rangle$ for $B,\langle\mathrm{R} 1, \mathrm{R} 2\rangle$ for $D$ - such that the respective factor is the only varying factor. Thus, interpreting one of these factors to be an effect of the underlying causal structure would contradict CA's homogeneity assumption. $C$ and $E$, thus, are the only potential effects of the causal structure generating table 3, i.e. $\mathrm{W}=\{C, E\}$. For each of the factors in $W$ minimally sufficient conditions are now identified. This is done in four steps: (1) a factor $Z_{i} \in \mathrm{W}$ is selected, (2) sufficient conditions of $Z_{i}$ are identified, (3) these sufficient conditions are minimalized, (4) the procedure is restarted at (1) by selecting another $Z_{j} \in \mathrm{W}$, until all factors in $\mathrm{W}$ have been selected. Let us take a detailed look at these four steps.

Step 1 - Selection of a potential effect: Randomly select one factor $Z_{i} \in \mathrm{W}$ such that $Z_{i}$ has not been selected in a previous run of steps 1 to 4 . $Z_{i}$ is termed effect ${ }^{*}$, the factors in $\left\{Z_{1}, \ldots, Z_{i-1}, Z_{i+1}, \ldots, Z_{n}\right\}$ are referred to as remainders. ${ }^{13}$

Step 2 - Identification of sufficient conditions: Identify all sufficient conditions of the effect* $Z_{i}$ according to the following rule:

(SUF) A coincidence $X_{k}$ of remainders is sufficient for $Z_{i}$ iff the input list $\mathcal{C}$ contains at least one row featuring $X_{k} Z_{i}$ and no row featuring $X_{k} \overline{Z_{i}}$.

The order of selecting effects* in step 1 does not matter, as long as it is guaranteed that eventually all members of W are selected. According to (SUF), a coincidence of remainders can only be sufficient for an effect* if it is instantiated at

\footnotetext{
${ }^{13}$ Selected factors are labelled effects $*$ to indicate that they possibly are the effects of the causal structure generating the input list. Effects* do not necessarily turn out to be (actual) effects of the underlying causal structure at the end of a CA-analysis. For instance, the set of effects* contained in list (d) of table 1 contains all factors in the frame - provided no further information is available that distinguishes among causes and effects. Yet, none of these effects* is identified as actual effects by CA.
} 
least once. Moreover, a coincidence of remainders contained in the input list is not sufficient for a selected effect* if it is also instantiated in combination with the absence of that effect*.

Let us perform these two steps on our example of table 3 by first selecting $C$ as effect*. Step 2 identifies three sufficient conditions of $C$, i.e. there are six coincidences of remainders that conform to (SUF): $A B D E, A B \bar{D} E, A \bar{B} D E, A \overline{B D} E$, $\bar{A} B D E, \bar{A} B \bar{D} E$. The first row (R1) of table 3 features the coincidence $A B D E$ in combination with $C$ and there is no row such that $A B D E$ is contained therein in combination with $\bar{C}$. $A B D E$, thus, is a sufficient condition of $C$ according to (SUF). Analogous considerations apply to the other sufficient conditions mentioned above: $\mathrm{R} 2$ is constituted by $A B \bar{D} E, \mathrm{R} 3$ by $A \bar{B} D E, \mathrm{R} 4$ by $A \overline{B D} E, \mathrm{R} 5$ by $\bar{A} B D E$, and R6 features $\bar{A} B \bar{D} E$ without either of these conditions being contained in combination with $\bar{C}$ in table 3 . Thus, each coincidence of remainders listed in the six rows featuring an instance of $C$ constitutes a sufficient condition of $C$.

Before sufficient conditions of the remaining effect* $E$ are identified, we proceed to minimalize the sufficient conditions of $C$.

Step 3 - Minimalization of sufficient conditions: The sufficient conditions of $Z_{i}$ identified in step 2 are minimalized according to the following rule:

(MSUF) A sufficient condition $Z_{1} Z_{2} \ldots Z_{h}$ of $Z_{i}$ is minimally sufficient iff neither $\overline{Z_{1}} Z_{2} \ldots Z_{h}$ nor $Z_{1} \overline{Z_{2}} \ldots Z_{h}$ nor $\ldots$ nor $Z_{1} Z_{2} \ldots \overline{Z_{h}}$ are sufficient for $Z_{i}$ according to (SUF).

Or operationally put:

(MSUF') Given a sufficient condition $Z_{1} Z_{2} \ldots Z_{h}$ of $Z_{i}$, for every $Z_{g} \in$ $\left\{Z_{1}, Z_{2}, \ldots, Z_{h}\right\}, h \geq g \geq 1$, and every $h$-tuple $\left\langle Z_{1^{\prime}}, Z_{2^{\prime}}, \ldots, Z_{h^{\prime}}\right\rangle$ which is a permutation of the $h$-tuple $\left\langle Z_{1}, Z_{2}, \ldots, Z_{h}\right\rangle$ : Eliminate $Z_{g}$ from $Z_{1} Z_{2} \ldots Z_{h}$ and check whether $Z_{1} \ldots Z_{g-1} Z_{g+1} \ldots Z_{h} \overline{Z_{i}}$ is contained in a row of $\mathcal{C}$. If that is the case, re-add $Z_{g}$ to $Z_{1} \ldots Z_{g-1} Z_{g+1} \ldots Z_{h}$ and eliminate $Z_{g+1}$; if that is not the case, proceed to eliminate $Z_{g+1}$ without re-adding $Z_{g}$. The result of performing this redundancy check on every factor contained in $Z_{1} Z_{2} \ldots Z_{h}$ is a set of minimally sufficient conditions of $Z_{i}$.

(MSUF) is nothing but an adaptation of the notion of a minimally sufficient condition as defined in section 2 to the context of coincidence lists. (MSUF'), on the other hand, can be seen as an operational expression of the analysans of the notion of a minimally sufficient condition implemented in (MSUF). That means, (MSUF) might be rephrased as follows: A sufficient condition $Z_{1} Z_{2} \ldots Z_{h}$ of $Z_{i}$ is minimally sufficient iff it results from an application of (MSUF'). At the price of high computational complexity, the formulation of (MSUF') is kept as simple as possible above. The order in which factors are eliminated from sufficient 
conditions matters as to the minimalization of such conditions - thus the systematic permutation of elimination orders. ${ }^{14}$ In many cases, however, it is not necessary to completely perform that permutation. For instance, assume an $h$-tuple $T_{1}=\left\langle Z_{1}, \ldots, Z_{d}, Z_{d+1}, \ldots, Z_{h}\right\rangle$ has been minimalized by means of (MSUF') up to element $Z_{d}$, that minimalization of $T_{1}$ can be taken over for all $h$-tuples $T_{2}=\left\langle Z_{1}, \ldots, Z_{d}, Z_{d+1^{\prime}}, \ldots, Z_{h^{\prime}}\right\rangle$ that coincide with $T_{1}$ up to element $Z_{d}$ without reapplying (MSUF') to $T_{2}$. Or suppose it has been found that $X_{1}=Z_{1} \ldots Z_{d}$ is a minimally sufficient condition of an investigated effect and a sufficient condition $X_{2}=Z_{1} Z_{2} \ldots Z_{h}$ containing $Z_{1} \ldots Z_{d}$ is to be minimalized by means of (MSUF'). In that case, it is not effective to minimalize $X_{2}$ by first eliminating the factors not contained in $X_{1}$, for this elimination order would just yield $X_{1}$ again.

Further optimizations of (MSUF') are conceivable, yet are not going to be discussed in the present context. More importantly, the intuition behind (MSUF') can be more colloquially captured: Every factor contained in a sufficient condition of $Z_{i}$ is to be tested for redundancy by eliminating it from that condition and checking whether the remaining condition still is sufficient for $Z_{i}$ or not. A sufficient condition of $Z_{i}$ is minimally sufficient iff every elimination of a factor from that condition results in the insufficiency of the remaining condition.

Performing step 3 on our exemplary case is straightforward. Step 2 yielded six sufficient conditions of $C$. For simplicity's sake, I only illustrate the minimalization of these six conditions by means of two examples. First, take $A B D E$. That this sufficient condition is not minimally sufficient for $C$ is seen by removing, say, $D$ and finding that $A B E$ itself is sufficient for $C$, for table 3 does not contain a row featuring $A B E \bar{C} . A B E$ still is not minimally sufficient. For instance, both $B$ and $E$ can be removed without sufficiency being lost. There is no row in 3 featuring $A \bar{C}$, which induces that $A$ is sufficient and, since it is a single factor that does not contain proper parts, minimally sufficient for $C$. There are other ways to further minimalize $A B E$ : A removal of $A$ and $E$ still yields a sufficient condition of $C$. There is no row in 3 featuring $B \bar{C}$. Therefore $B$ is minimally sufficient for $C$. Second, let us look at the second sufficient condition of $C$ identified by (SUF). $A B \bar{D} E$ is not minimally sufficient because $A B$ can be removed without sufficiency for $C$ being lost. There is no row in 3 featuring $\overline{C D} E$, which induces that $\bar{D} E$ is sufficient for $C$. If $\bar{D} E$ is further reduced, sufficiency is lost. R7 features $\bar{C} E$ and R8 $\overline{C D}$, which amounts to neither $E$ nor $\bar{D}$ being sufficient for $C . \bar{D} E$, hence, is minimally sufficient for $C$. Minimalizing the other sufficient conditions of $C$ by analogously implementing (MSUF') does not yield any further minimally sufficient conditions. All in all, therefore, minimalizing the sufficient conditions of $C$ generates the following three minimally sufficient conditions: $A, B$, and $\bar{D} E$.

After having identified the minimally sufficient conditions of a first factor $Z_{i} \in \mathrm{W}$, the same needs to be done for all other effects*. We thus need a loop

\footnotetext{
${ }^{14}$ This is an important deviation from the minimalization of sufficient conditions as performed by $Q C A$. $Q C A$ only eliminates conjuncts of a sufficient condition if the latter reduced by the respective conjunct is actually contained in the coincidence list. I take this restriction to be a serious limitation of the minimizability of sufficient conditions.
} 
that brings CA back to step 1 , if not all factors in $\mathrm{W}$ have been assigned minimally sufficient conditions yet.

Step 4-(MSUF)-Loop: If all $Z_{i} \in \mathrm{W}$ have been selected as effects* proceed to step 5, otherwise go back to step 1 .

Applying this loop to our example yields seven sufficient conditions of $E$. Each row featuring $E$ comprises a sufficient condition of remainders: $A B C D, A B C \bar{D}$, $A \bar{B} C D, A \bar{B} C \bar{D}, \bar{A} B C D, \bar{A} B C \bar{D}, \overline{A B C} D$. For example, $\mathrm{R} 2$ of table 3 is constituted by $A B C \bar{D}$ and there is no row featuring $A B C \bar{D}$ along with $\bar{E}$, or R3 comprises $A \bar{B} C D$ and no row in 3 contains $A \bar{B} C D$ in combination with $\bar{E}$. The sufficiency of the other conditions is analogously demonstrated. Employing (MSUF) or (MSUF') to minimalize these conditions brings forth four minimally sufficient conditions of $E: A, B, C$, and $D$. The list in table 3 contains no rows featuring either $A \bar{E}, B \bar{E}, C \bar{E}$, or $D \bar{E}$.

As an overall result of performing the first stage (steps 1 to 4 ) of CA on our exemplary case, we have thus identified the following minimally sufficient conditions of the factors in $\mathrm{W}$.

Result after 4 steps:
\begin{tabular}{|c|l|}
\hline$Z_{i} \in \mathrm{W}$ & $\begin{array}{l}\text { minimally sufficient } \\
\text { conditions }\end{array}$ \\
\hline$C$ & $A, B, \bar{D} E$ \\
$E$ & $A, B, C, D$ \\
\hline
\end{tabular}

\section{Identification and Minimalization of Necessary Conditions}

As the famous Manchester Hooters counterexample against Mackie's (1974) INUS-theory of causation ${ }^{15}$ demonstrates and as articulated in our analysis of causal relevance given in section 2, minimally sufficient conditions are not generally causally interpretable. Only minimally sufficient conditions that are moreover non-redundant parts of minimally necessary conditions are amenable to a causal interpretation. ${ }^{16}$ After having identified minimally sufficient conditions, we thus now proceed to first form necessary conditions of the effects* from their minimally sufficient conditions and then minimalize these necessary conditions. Since factor frames processed by CA are incomplete with respect to underlying causal structures, i.e. there supposedly will always be many causally relevant factors not contained in input lists, effects* can only be assigned necessary conditions relative to the homogeneous backgrounds of corresponding coincidence lists. This is easily accomplished by disjunctively combining the minimally sufficient conditions

\footnotetext{
${ }^{15}$ Cf. Mackie (1974), Baumgartner and Graßhoff (2004), ch. 5.

${ }^{16}$ How a minimalization of necessary conditions solves the Hooters-Problem is illustrated in section 10 .
} 
of each effect*, yielding one necessary condition relative to an input list $\mathcal{C}$ and its background for each factor $Z_{i} \in \mathrm{W}$.

Step 5 - Identification of necessary conditions: Identify a necessary condition of each effect* $Z_{i}$ by disjunctively concatenating $Z_{i}$ 's minimally sufficient conditions according to the following rule:

(NEC) A disjunction $X_{1} \vee X_{2} \vee \ldots \vee X_{h}$ of minimally sufficient conditions of $Z_{i}$ is necessary for $Z_{i}$ iff $\mathcal{C}$ contains no row featuring $Z_{i}$ in combination with $\neg\left(X_{1} \vee X_{2} \vee \ldots \vee X_{h}\right)$, i.e. no row comprising $\overline{X_{1} X_{2} \ldots X_{h}} Z_{i}$.

Performed on our example, step 5 issues $A \vee B \vee \bar{D} E$ and $A \vee B \vee C \vee D$ as necessary conditions of $C$ and $E$, respectively. That means, there is no row in 3 featuring $C$ in combination with neither $A$ nor $B$ nor $\bar{D} E$. Whenever $C$ is instantiated, there is also an instance of at least one of its minimally sufficient conditions. Similarly for $E$ : No row of 3 records a coincidence of $E$ with neither an instance of $A$ nor $B$ nor $C$ nor $D$. $E$ is always instantiated in combination with one of its minimally sufficient conditions.

Such as to determine whether the minimally sufficient conditions assigned to the effects* at the end of the previous section in fact are non-redundant parts of necessary conditions, these necessary conditions have to be minimalized.

Step 6-Minimalization of necessary conditions: The necessary conditions of every $Z_{i} \in \mathrm{W}$ identified in step 5 are minimalized according to the following rule:

(MNEC) A necessary condition $X_{1} \vee X_{2} \vee \ldots \vee X_{h}$ of $Z_{i}$ is minimally necessary iff neither $X_{2} \vee X_{3} \vee \ldots X_{h}$ nor $X_{1} \vee X_{3} \vee \ldots X_{h}$ nor ... nor $X_{1} \vee$ $X_{2} \vee \ldots \vee X_{h-1}$ is necessary for $Z_{i}$ according to (NEC).

Or operationally put:

(MNEC') Given a necessary condition $X_{1} \vee X_{2} \vee \ldots \vee X_{h}$ of $Z_{i}$, for every $X_{g} \in$ $\left\{X_{1}, X_{2}, \ldots, X_{h}\right\}, h \geq g \geq 1$, and every $h$-tuple $\left\langle X_{1^{\prime}}, X_{2^{\prime}}, \ldots, X_{h^{\prime}}\right\rangle$ which is a permutation of the $h$-tuple $\left\langle X_{1}, X_{2}, \ldots, X_{h}\right\rangle$ : Eliminate $X_{g}$ from $X_{1} \vee X_{2} \vee \ldots \vee X_{h}$ and check whether there is a row in $\mathcal{C}$ featuring $Z_{i}$ in combination with $\neg\left(X_{1} \vee \ldots \vee X_{g-1} \vee X_{g+1} \vee \ldots \vee X_{h}\right)$, i.e. a row comprising $\overline{X_{1} \ldots X_{g-1} X_{g+1} \ldots X_{h}} Z_{i}$. If that is the case, re-add $X_{g}$ to $X_{1} \vee \ldots \vee X_{g-1} \vee X_{g+1} \vee \ldots \vee X_{h}$ and eliminate $X_{g+1}$; if that is not the case, proceed to eliminate $X_{g+1}$ without re-adding $X_{g}$. The result of performing this redundancy check on every minimally sufficient condition contained in $X_{1} \vee X_{2} \vee \ldots \ldots \vee X_{h}$ is a set of minimally necessary conditions of $Z_{i}$.

In analogy to (MSUF), (MNEC) is nothing but an adaptation of the notion of a minimally necessary condition as defined in section 2 to the context of coincidence lists. (MNEC'), on the other hand, can be seen as an operational expression of 
the analysans of the notion of a minimally necessary condition implemented in (MNEC). That means, (MNEC) might be rephrased as follows: A necessary condition $X_{1} \vee X_{2} \vee \ldots \vee X_{h}$ is minimally necessary iff it results from an application of (MNEC'). The formulation of (MNEC') has been kept as simple as possible at the expense of its computational complexity. Analogous optimizations as in case of $(\text { MSUF' })^{17}$ are possible with respect to (MNEC'). The intuition behind (MNEC') can also be more colloquially captured: Every minimally sufficient condition contained in a necessary condition of $Z_{i}$ is to be tested for redundancy by eliminating it from that condition and checking whether the remaining condition still is necessary for $Z_{i}$ or not. A necessary condition of $Z_{i}$ is minimally necessary iff every elimination of a minimally sufficient condition from that necessary condition results in the loss of necessity of the remaining condition.

Let us illustrate the minimalization of necessary conditions by first performing step 6 on the necessary condition $A \vee B \vee \bar{D} E$ of $C$. That disjunction is not minimally necessary for $C$, because it contains a necessary proper part: $A \vee B$. Whenever $C$ is instantiated in table 3 , there is an instance of either $A$ or $B .3$ does not contain a row featuring $\overline{A B} C . \bar{D} E$ does not amount to a non-redundant part of a minimally necessary condition, for whenever $\bar{D} E$ is instantiated in combination with $C$, there also is an instance of $A \vee B$. The same results from applying (MNEC') to $A \vee B \vee \bar{D} E$. Upon eliminating $A$ we find that the rest is no longer necessary for $C$, because R3 of table 3 features $\overline{B \bar{D}} E C$, or more specifically $\bar{B} D E C$. Hence, $A$ is re-added. The same is found upon removing $B$. R5 features $\overline{A \bar{D}} E C$ or $\bar{A} D E C$, respectively. Removing $\bar{D} E$, however, does not result in a loss of necessity. Therefore, $\bar{D} E$ is not re-added. $A \vee B \vee C \vee D$ neither amounts to a minimally necessary condition of $E . A \vee B \vee C \vee D$ not only contains one but two necessary proper parts: $C \vee D$ and $A \vee B \vee D$. There is no row in 3 featuring $\overline{C D} E$ or $\overline{A B D} E$. Whenever $E$ is instantiated, there is an instance of $C \vee D$ and of $A \vee B \vee D$. These two ways to minimalize $A \vee B \vee C \vee D$ stem from the fact that there are biconditional dependencies among the minimally sufficient conditions of $E$. Within the homogeneous background of table 3, $C$ is instantiated if and only if $A \vee B$ is instantiated.

Result after 6 steps:
\begin{tabular}{|c|l|}
\hline$Z_{i} \in \mathrm{W}$ & $\begin{array}{l}\text { minimally necessary } \\
\text { conditions }\end{array}$ \\
\hline$C$ & $A \vee B$ \\
$E$ & $A \vee B \vee D, C \vee D$ \\
\hline
\end{tabular}

\section{Framing Minimal Theories}

Step 6 of CA yields a set of minimally necessary disjunctions of minimally sufficient conditions for each $Z_{i} \in \mathrm{W}$. We have thus come close to assigning a minimal

\footnotetext{
${ }^{17}$ Cf. p. 16 above.
} 
theory to the list in table 3 . The result of step 6 allows for framing one simple minimal theory for $C$ and two for $E$. Relative to the homogeneous background of table 3 , these minimal theories can be straightforwardly expressed thus: $A \vee B \Rightarrow C$, $A \vee B \vee D \Rightarrow E, C \vee D \Rightarrow E$. However, apart from the specific causal background of table 3, it must not be the case that $A$ and $B$ are themselves sufficient for $C$ or $C$ and $D$ for $E$. Moreover, there may well be further minimally sufficient conditions of both $C$ and $E$. Therefore, suspending the relativization to the background of 3 and expressing these dependencies in their general and background independent form leads to:

$$
\begin{aligned}
A X_{1} \vee B X_{2} \vee Y_{C} & \Rightarrow C \\
A X_{1} \vee B X_{2} \vee D X_{3} \vee Y_{E} & \Rightarrow E \\
C X_{1} \vee D X_{2} \vee Y_{E} & \Rightarrow E
\end{aligned}
$$

$C$ and $E$ have a non-empty intersection of minimally sufficient conditions. Correspondingly, the simple minimal theories of $C$ and $E$ share a number of common factors. The causal structure regulating the behavior of $E$ is not independent of the structure behind the behavior of $C$. The behavior of the factors in table 3 , thus, is regulated by a complex causal structure. In order to determine what that structure looks like, the simple minimal theories of $C$ and $E$ are to be conjunctively combined to form a complex theory. Here an interesting ambiguity emerges: (8) and (9) - if causally interpreted - identify different direct causal relevancies for $E$. While according to (8) $A$ and $B$ are directly causally relevant to $E$, (9) instead holds $C$ to be directly relevant to $E$. The coincidences in table 3 are either generated by a causal chain, such that $A$ and $B$ are parts of alternative causes of $C$ while $C$ and $D$ are contained in alternative causes of $E$, or they are generated by an epiphenomenon, such that $A$ and $B$ are parts of alternative causes of $C$ while $A, B$, and $D$ are contained in alternative causes of $E$. The two causal structures possibly underlying the list in table 3 are graphed in figure 2. Thus, the minimalization of $E$ 's necessary condition is ambiguous. ${ }^{18}$

The list in table 3 is underdetermined as to whether its coincidences are the result of a chain or an epiphenomenon. This is not a specific anomaly of list 3 . Rather, to any causal chain there exists an epiphenomenon that generates the same coincidence list as the chain - accordingly, call the chain and the corresponding epiphenomenon $c$-equivalent causal structures. That means, any coincidence list that could be the result of a chain could also be the result of $c$-equivalent epiphenomenon. ${ }^{19}$

Epiphenomena with a coincidence frame matching the coincidence frame of a causal chain have a very specific structure: All factors that are part of a minimally

\footnotetext{
${ }^{18}$ That the minimalization of necessary conditions can be ambiguous is not taken into account in the context of $Q C A$. Both in Ragin (1987) and Ragin (2000) the minimalization of necessary conditions is assumed to be unproblematic. For another ambiguity with respect to minimalizing necessary conditions cf. Quine (1959) and Kim (1993).

${ }^{19}$ For a proof of this general underdetermination of lists that are compatible with causal chains cf. Baumgartner (forthcoming), ch. 4.
} 


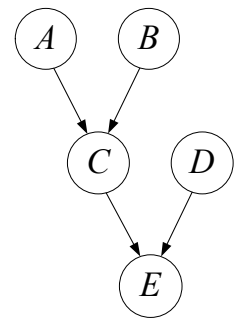

(a)

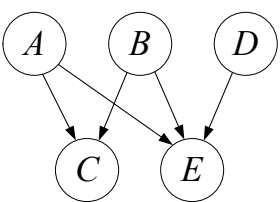

(b)

Fig. 2: A causal chain and an epiphenomenon that both could underly the coincidences in list 3 .

sufficient condition of one effect - e.g. $C$ in graph (b) of fig. 2 - are also contained in the minimally sufficient conditions of the other effect $-E$ in case of (b). Two factors satisfying this structural pattern such as $C$ and $E$ shall be referred to as entangled factors. Entangled factors are not only contained in epiphenomena as (b), but notably in chains. $C$ and $E$ are entangled in graph (a) of figure 2 as well. Two factors are entangled iff every conjunct of a minimally sufficient condition of one of the two factors is also a conjunct of at least one minimally sufficient condition of the other factor.

Every epiphenomenon that shares its coincidence frame with a causal chain features at least two entangled factors. That means, at most one effect of such an epiphenomenon has causally relevant factors that are not part of a minimally sufficient condition of the other effect. For the purpose of an easy reference to such epiphenomenal structures, I shall in this context speak of entangled epiphenomena.

There thus exists an entangled epiphenomenon to every causal chain such that the epiphenomenon and the chain generate the same coincidence list. The converse, however, does not hold. The epiphenomenon (b) of figure 1, for instance, which is not entangled, is easily distinguishable from a chain as (a). The causal structure behind the behavior of two factors $A$ and $B$ cannot be a chain if the following conditions hold: Among the minimally sufficient conditions of $A$ there is a condition containing at least one factor $Z_{1}$ which is not part of any minimally sufficient condition of $B$; and among the minimally sufficient conditions of $B$ there is a condition containing at least one factor $Z_{2}$ which is not part of any minimally sufficient condition of $A$. If $A$ and $B$, furthermore, have at least one minimally sufficient condition in common, their behavior must be regulated by an epiphenomenal structure. All of these 'ordinary' epiphenomena are identifiable via the coincidences they generate.

As long as causal inference methodologies exclusively analyze coincidence information, an unambiguous inference to causal chains is excluded in principle. Based on such pure coincidence analyses every causal process which is commonly assumed to be structured in terms of a chain could just as well be modeled as an entangled epiphenomenon. This, of course, is a finding that heavily conflicts with common intuitions. The fact that causes and effects are ordinarily concatenated in 
chains is one of the core features of our understanding of the causal relation. The inability of pure coincidence analyses to distinguish between chains and epiphenomena thus is a serious problem. I call it the chain-problem.

Several solutions to the chain-problem can be thought of. The chain-problem might be taken to demonstrate that apart from coincidence information inferring complex structures requires further empirical information as e.g. a temporal ordering of the instances of causally related factors or information as to the direction of energy transfer among these instances. In Baumgartner (forthcoming) (ch. 4), however, I show that such further empirical information merely narrows down the amount of epiphenomena that are $c$-equivalent to chains, but does not prevent there being a $c$-equivalent epiphenomenon to every chain. The chain-problem cannot be solved on purely empirical grounds. Rather, it must be solved on pragmatic or conventional grounds. Modelling the behaviour of the factors in table 1 in terms of a chain or an epiphenomenon is empirically equivalent, but the two causal models highly differ with respect to explanatory power and empirical content. First, modelling the structure behind table 1 in virtue of a chain explains the entanglement of $C$ and $E$. If $C$ is determined to be sufficient for $E$, it immediately follows from the transitivity of sufficiency that whatever is sufficient for $C$ is also sufficient for $E$. Thus, the entanglement of $C$ and $E$ is a structural necessity of the chain model. Moreover, modelling the behaviour of the factors in table 1 in terms of a chain stipulates that the entanglement of $C$ and $E$ will subsist regardless of what other factors are subsequently identified as further causes of $C$ or $E$. The epiphenomenal model, on the other hand, not only fails to explain why $C$ should be entangled with $E$, it furthermore is completely indifferent as to the subsistence of this entanglement across later expansions of the model. This is why preference shall always be given to a causal interpretation of entanglements, i.e. to the chain model. Accordingly, the causal structure behind coincidence lists that could stem both from a chain and an entangled epiphenomenon shall always be modelled in terms of a chain. ${ }^{20}$

That means, complex minimal theories are to be built up from simple theories such that for every $i, 1 \leq i<n$, in a sequence of entangled factors $Z_{1}, \ldots, Z_{n}$, $n \geq 2: Z_{i}$ is contained in the antecedent of the simple minimal theory of $Z_{i+1}$. Hence, of (10) and (11) only (10) constitutes a complex minimal theory.

$$
\begin{array}{r}
(A \vee B \Rightarrow C) \wedge(C \vee D \Rightarrow E) \\
\left(A X_{1} \vee B X_{2} \vee Y_{C} \Rightarrow C\right) \wedge\left(C X_{3} \vee D X_{4} \vee Y_{E} \Rightarrow E\right) \\
(A \vee B \Rightarrow C) \wedge(A \vee B \vee D \Rightarrow E) \\
\left(A X_{1} \vee B X_{2} \vee Y_{C} \Rightarrow C\right) \wedge\left(A X_{1} X_{3} \vee B X_{2} X_{3} \vee Y_{C} X_{3} \vee D X_{4} \vee Y_{E} \Rightarrow E\right)
\end{array}
$$

\footnotetext{
${ }^{20}$ For a detailed discussion of the chain-problem and its solution cf. Baumgartner (forthcoming), ch. 4 .
} 
All in all, in the remaining step of CA minimal theories are framed from the minimally necessary disjunctions of minimally sufficient conditions identified for each $Z_{i} \in \mathrm{W}$ in step 6 . This is done by means of a twofold procedure:

Step 7 - Framing minimal theories: The minimally necessary disjunctions of minimally sufficient conditions of each $Z_{i} \in \mathrm{W}$ identified in step 6 are assembled to minimal theories as follows:

(1) For each $Z_{i} \in \mathrm{W}$ and each minimally necessary disjunction $X_{1} \vee X_{2} \vee$ $\ldots \vee X_{h}, h \geq 2,{ }^{21}$ of minimally sufficient conditions of $Z_{i}$ : form a simple minimal theory $\Psi$ of $Z_{i}$ by making $X_{1} \vee X_{2} \vee \ldots \vee X_{h}$ the antecedent of a double-conditional and $Z_{i}$ its consequent: $X_{1} \vee X_{2} \vee$ $\ldots \vee X_{h} \Rightarrow Z_{i}$.

(2) Conjunctively combine two simple minimal theories $\Phi$ of $Z_{i}$ and $\Psi$ of $Z_{j}$ to the complex minimal theory $\Phi \wedge \Psi$ iff $\Phi$ and $\Psi$ conform to the following conditions:

(a) at least one factor in $\Phi$ is part of $\Psi$;

(b) $\Phi$ and $\Psi$ do not have an identical consequent;

(c) for every $i, 1 \leq i<n$, in a sequence of entangled factors $Z_{1}$, $\ldots, Z_{n}, n \geq 2: Z_{i}$ is contained in the antecedent of the simple minimal theory of $Z_{i+1}$.

Applied to our example, step 7 assigns the following complex and background independent minimal theory to the coincidence list in table 3 :

Result after 7 steps:

\begin{tabular}{|c|l|}
\hline$Z_{i} \in \mathrm{W}$ & minimal theory \\
\hline$C$ & $\left(A X_{1} \vee B X_{2} \vee Y_{C} \Rightarrow C\right) \wedge\left(C X_{3} \vee D X_{4} \vee Y_{E} \Rightarrow E\right)$ \\
$E$ & \\
\hline
\end{tabular}

\section{Causal Interpretation}

After having assigned a minimal theory to a coincidence list, the by far most intricate hurdles on the way to uncovering the causal structure behind that list have been overcome. As we have seen in section 2, there exists a straightforward syntactical convention as regards the causal interpretation of minimal theories. Minimal theories render causal structures syntactically transparent:

Step $8^{*}$-Causal interpretation: Disjuncts in the antecedent of simple minimal theories are to be interpreted as alternative (complex) causes of the factor

\footnotetext{
${ }^{21}$ The constraint as to a minimum of two alternative minimally sufficient conditions for each effect* does justice to the minimal complexity of a causal structure required such that its direction is identifiable (cf. section 2).
} 
in the consequent. Conjuncts constituting such disjuncts correspond to nonredundant parts of complex causes. Triples of factors $\left\langle Z_{h}, Z_{i}, Z_{j}\right\rangle$, such that $Z_{h}$ appears in the antecedent of a minimal theory of $Z_{i}$ and $Z_{i}$ is part of a minimal theory of $Z_{j}$, are to be interpreted as causal chains.

This interpretation rule is not to be seen as part of CA proper. Nonetheless, it fulfills an essential function on the way to a causal inference. For this reason, the rule concerning causal interpretation is starred.

CA thus determines the coincidences in our exemplary table 3 to be the result of a causal chain: $A$ and $B$ are parts of alternative causes of $C$ while $C$ and $D$ are contained in alternative causes of $E$. Thereby, $A$ and $B$ are moreover rendered indirectly causally relevant to $E$. Steps $0^{*}$ to 7 assign a minimal theory to a coincidence list and step $8 *$ causally interprets that theory.

\section{A Further Example}

After having completely laid out CA and after having tested its performance with respect to a first concrete coincidence list, CA shall now be applied to another example. Consider the list in table 4. It covers the same factor frame as table 3 and only differs from the latter as regards to four rows: R2, R4, R6, R7. In order to determine the set $\mathrm{W}$ of potential effects, it again is assumed that no factor in $\{A, B, C, D, E\}$ is excluded from effect position by prior causal knowledge or spatiotemporal constraints. For reasons of compatibility with (HC), however, factors $A, B$, and $C$ cannot be effects. For each of these factors there is a pair of rows in table $4-\langle\mathrm{R} 1, \mathrm{R} 5\rangle$ for $A,\langle\mathrm{R} 1, \mathrm{R} 3\rangle$ for $B,\langle\mathrm{R} 1, \mathrm{R} 2\rangle$ for $C$ - such that the respective factor is the only varying factor. $D$ and $E$, thus, are the potential effects of the causal structure generating table 4 , i.e. $\mathrm{W}=\{D, E\}$.

Performing steps 2 and 3 on $D$ and $E$ yields the following:

Sufficient conditions of $D$ : $A B C E, A B \bar{C} E, A \bar{B} C E, A \overline{B C E}, \bar{A} B C E, \bar{A} B \bar{C} E$.

\begin{tabular}{ccccc}
$A$ & $B$ & $C$ & $D$ & $E$ \\
\hline 1 & 1 & 1 & 1 & 1 \\
1 & 1 & 0 & 1 & 1 \\
1 & 0 & 1 & 1 & 1 \\
1 & 0 & 0 & 1 & 0 \\
0 & 1 & 1 & 1 & 1 \\
0 & 1 & 0 & 1 & 1 \\
0 & 0 & 1 & 0 & 1 \\
0 & 0 & 0 & 0 & 0
\end{tabular}

Tab. 4: A second exemplary coincidence list over the same factor frame as the list in table 3. 
Minimally sufficient conditions of $D: A, B, \bar{C} E$.

Sufficient conditions of $E: A B C D, A B \bar{C} D, A \bar{B} C D, \bar{A} B C D, \bar{A} B \bar{C} D$, $\overline{A B} C \bar{D}$.

Minimally sufficient conditions of $E: B, C, \bar{A} D$.

After having identified minimally sufficient conditions, CA proceeds to first form and then minimalize necessary conditions for each effect* ${ }^{*}$.

Necessary condition of $D: A \vee B \vee \bar{C} E$.

Minimally necessary condition of $D: A \vee B$.

Necessary condition of $E: B \vee C \vee \bar{A} D$.

Minimally necessary condition of $E: B \vee C$.

The minimally sufficient conditions $\bar{C} E$ of $D$ and $\bar{A} D$ of $E$ are not part of minimally necessary conditions of $D$ and $E$, for whenever they are instantiated, there is an instance of another disjunct in the corresponding necessary conditions. The two conditions thus are redundant within their necessary conditions. Finally, CA frames one simple minimal theory for $D$ and $E$ each:

$$
\begin{aligned}
& A X_{1} \vee B X_{2} \vee Y_{D} \Rightarrow D \\
& B X_{3} \vee C X_{4} \vee Y_{E} \Rightarrow E
\end{aligned}
$$

(12) and (13) have one factor in common - $B$ - while none are entangled. Hence, the two simple minimal theories can unambiguously be conjunctively joined to constitute a complex theory representing the causal structure generating table 4 .

$$
\left(A X_{1} \vee B X_{2} \vee Y_{D} \Rightarrow D\right) \wedge\left(B X_{3} \vee C X_{4} \vee Y_{E} \Rightarrow E\right)
$$

Accordingly, this input list is the result of an epiphenomenon such that $B$ is the common cause of $D$ and $E$.

It should be pointed out that this epiphenomenal structure coincides with the causal structure behind Mackie's Manchester Factory Hooters. Mackie (1974) cannot adequately account for such epiphenomenal structures by means of his INUStheory of causation. This defect of the INUS-theory stems from the fact that Mackie does not minimalize necessary conditions. The example in table 4 now shows that minimalizing necessary conditions in terms of (MNEC) and (MNEC'), respectively, solves the Hooters-Problem, as it makes structures of the Hooters kind amenable to an adequate causal interpretation. This finding demonstrates that epiphenomenal structures are properly causally analyzable on regularity theoretic grounds - notwithstanding the widespread opinion to the contrary among critics of regularity accounts. 


\section{Summary}

This paper has introduced a procedure of causal reasoning embedded in the regularity theoretic framework and mainly implementing Boolean techniques. Coincidence analysis (CA) differs from $Q C A$ essentially in three respects: First, CA does not assume there to be a singular effect in every causally analyzed factor frame, second CA does not presuppose the mutual independence of the causes of that effect, and third, as to CA it must not be known prior to applying CA what factor within the analyzed frame is the effect and, accordingly, what factors are possible causes. Thus, CA abandons the three $Q C A$-assumptions (SNG), (IND), and (ICE). We have shown that these causal assumptions made in the context of $Q C A$ are not indispensable for causal inferences drawn from mere coincidence information. Thus, homogeneity (HC) turns out to be the only causal assumption needed for causal reasoning based on pure coincidence data.

As an immediate consequence thereof, CA is not limited to uncovering causal structures layer by layer. While $Q C A$ is only applicable provided that prior causal knowledge separates analyzed factor frames in a subset consisting of causally independent (possible) cause factors and a subset consisting of a single effect, CA is applicable even without any prior causal knowledge concerning the underlying structure. CA is capable of analyzing causal structures from scratch and in their whole complexity. Due to limited space, of course, the two examples discussed here are simple and purposefully tailored thus that the performance of CA with respect to the two complex causal structures that are critical for $Q C A$ is illustrated. In principle, however, CA is capable of analyzing structures of arbitrary complexity. Considerably more complex examples can be found in Baumgartner (forthcoming).

Apart from generalizing $Q C A$, CA fills a gap left open by the probabilistic algorithms of causal reasoning as presented in Spirtes, Glymour and Scheines (2000 (1993)). These algorithms only generate informative outputs provided that analyzed conditional probabilities are lower than 1, i.e. provided that causes do not in a strict sense determine their effects. CA, thereagainst, is custom-built to deterministic causal dependencies and properly uncovers such dependencies.

As shown in sections 3 and 4, not every coincidence list is causally analyzable. Accordingly, CA cannot be seen as a complete inference procedure in the sense that it assigns a causal structure to a coincidence list whenever the coincidences in that list are in fact the result of such a structure. Empirical data may be insufficient to uncover its causal regularities. However, CA is a correct causal inference procedure in the sense that whenever CA assigns a causal structure to a coincidence list, that list is in fact generated by that structure. CA unambiguously assigns a minimal theory and thereby a causal structure to every causally interpretable coincidence list. 


\section{References}

Baumgartner, Michael and Graßhoff, Gerd: Kausalität und kausales Schliessen. Eine Einführung mit interaktiven Übungen, Bern: Bern Studies 2004.

Baumgartner, Michael: Complex Causal Structures. Extensions of a Regularity Theory of Causation, forthcoming.

Broad, Charles D.: The Principles of Demonstrative Induction I-II, Mind, 39 (1930), 302 317, 426-439.

- Hr. von Wright on the Logic of Induction I-III, Mind, 53 (1944), 1-24, 97-119, 193-214.

Ehring, Douglas: Causal Asymmetry, The Journal of Philosophy, 79 (1982), 761-774.

Graßhoff, Gerd and May, Michael: Causal Regularities, In Spohn, W., Ledwig, M. and Esfeld, M., editors: Current Issues in Causation, Paderborn: Mentis 2001, 85-114.

Hausman, Daniel: Causal Asymmetries, Cambridge: Cambridge University Press 1998.

Kim, Jaegwon: Causes and Events: Mackie on Causation, In Sosa, Ernest and Tooley, Michael, editors: Causation, Oxford: Oxford University Press 1993, 60-74.

Mackie, John L.: The Cement of the Universe. A Study of Causation, Oxford: Clarendon Press 1974.

Quine, Willard van Orman: On Core and Prime Implicants of Truth Functions, American Mathematical Monthly, 66 (1959), 755-760.

Ragin, Charles C.: The Comparative Method, Berkeley: University of California Press 1987.

Fuzzy-Set Social Science, Chicago: University of Chicago Press 2000.

Sanford, David: The Direction of Causation and the Direction of Conditionship, The Journal of Philosophy, 73 (1976), 193-207.

Spirtes, Peter, Glymour, Clark and Scheines, Richard: Causation, Prediction, and Search, 2nd edition. Cambridge: MIT Press 2000 (1993). 